\title{
Fünf Jahre Psychotherapeutengesetz
}

Am 1. Januar 1999 trat das Psychotherapeutengesetz in Kraft. Psychotherapeutinnen und Psychotherapeuten erscheinen zumindest aus dem Inneren betrachtet - oft als eine Berufsgruppe, die sich durch unzählige Organisationen und Verbände mit unterschiedlichen, oft konträren Interessen und Anliegen gegenseitig «das Wasser abgräbt». Seit den 1970er Jahren haben wir es «geschafft», durch Pflege und Betonung dieser Unterschiede und durch einen Mangel an Kommunikationsbereitschaft und Kompromissfähigkeit eine gesetzliche Grundlage für einen akademischen Heilberuf etwa 20 Jahre lang mit zu verhindern. Groß waren die Klüfte und überaus ambitioniert die Forderungen der einzelnen Gruppen. Nur - sie passten nicht in die politische Landschaft, und durch die Uneinigkeit konnten lediglich Not- und Übergangslösungen, beispielsweise das Delegationsverfahren, die therapeutische Arbeit auf der Basis einer eingeschränkten Heilpraktikererlaubnis oder die so genannte TK-Regelung, geschaffen werden.

Die Profession der Psychologischen Psychotherapeuten und der Kinder- und Jugendlichenpsychotherapeuten ist auch wie kaum eine zweite - durch unterschiedliche Modellannahmen zur Ätiologie und Behandlung der relevanten Störungen bzw. Erkrankungen geprägt. Der Schulenstreit beherbergte dabei die Diskussion um die «Wahrheit» dieser Vorstellungen, um die Ursachen, die Entwicklung und die Behandlung von Störungen: Sind psychische Störungen erlernt? Sind sie eine Folge frühkindlicher Konflikte zwischen Ich und Es? Sind sie dadurch zu erklären, dass das System Familie nicht richtig funktioniert, oder sind sie Ausdruck einer ungenügenden Selbstaktualisierung? Kann eine Behandlung genügen, die «bloß» das Symptom kuriert, oder führt diese «oberflächliche» Behandlung unweigerlich zu unheilvollen Symptomverschiebungen, weil die in der Lebensgeschichte liegenden Ursachen und die «Persönlichkeit» nicht durch eine Reaktualisierung kindlich-interaktiver Beziehungen behandelt wurden? Reicht eine unspezifische Behandlung, bei der «Echtheit» der
Beziehung zwischen Therapeut und Klient die Selbstaktualisierung verbessert? Oder kann man durch zirkuläres Fragen ein «pathologisches» Äquilibrium in einem System so verändern, dass die Störung des Systems zu einer Heilung des «Symptomträgers» führt?

Bei vielen Auseinandersetzungen in der Entstehungsgeschichte des Psychotherapeutengesetzes war der Wunsch, mit den jeweils eigenen Vorstellungen «Recht» zu bekommen, ein starker Motor. Anderes «Futter» bekamen die Auseinandersetzungen durch grundsätzlich unterschiedliche Krankheitsvorstellungen: eher biologisch-medizinisch oder eher psychologisch-sozial. Da diese wiederum oft mit den häufigsten Grundberufen der Profession - Medizin oder Psychologie verschränkt waren, spielten hier auch berufspolitische Auseinandersetzungen um den «adäquaten» Grundberuf und die Verteilung von Zuständigkeiten und Zugangsmöglichkeiten zu den sozialrechtlich geregelten Töpfen der Gesetzlichen Krankenkassen eine Rolle.

Zwar sind diese Motive und die dadurch entstandene Dynamik durch die Verabschiedung des Psychotherapeutengesetzes nicht versiegt oder kraftlos geworden; nach wie vor gibt es fachlich und berufsständisch motivierte Auseinandersetzungen zwischen den verschiedenen Gruppen. Von außen betrachtet hat der Gesetzgeber jedoch einen geschickten (systemischen?) Zug getan: Er hat die Psychologischen Psychotherapeuten in einigen der neu gebildeten Institutionen, aber auch die verschiedenen Grundberufe gleich behandelt und in gleiche Gremien gesteckt. Er hat sich aus berufsständischen und schulenspezifischen Disputen herausgehalten und diese, vornehmlich durch die Einsetzung eines Wissenschaftlichen Beirats und durch die Anpassungen der Heilberufe-Kammergesetze auf Landesebene, der Profession selbst überlassen. Hier sollen sich die Fachleute auseinandersetzen und $\mathrm{zu}$ einem gemeinsamen Urteil über die Wissenschaftlichkeit der psychotherapeutischen Verfahren, die Sicherung der Qualität

\begin{tabular}{ll}
\hline KARGER & ( ) 2004 S. Karger GmbH, Freiburg \\
$\begin{array}{l}\text { Fax +497614520714 } \\
\begin{array}{l}\text { E-mail Information@Karger.de } \\
\text { www.karger.com }\end{array}\end{array}$ & $\begin{array}{l}\text { Accessible online at: } \\
\text { www.karger.com/ver }\end{array}$ \\
&
\end{tabular}

Prof. Dr. Thomas Fydrich

ZPP - Zentrum für Psychologische Psychotherapie

Psychologisches Institut der Universität

Hauptstr. 51, D-69117 Heidelberg

Tel. +49 6221 547-292, Fax -600

E-mail fydrich@psychologie.uni-heidelberg.de 
der Versorgung und die Umsetzung beruflicher Standards kommen; unabhängig von historischen, erkenntnistheoretischen und praktischen Wurzeln der verschiedenen therapeutischen Ansätze; möglicherweise jedoch unter Berücksichtigung dieser.

Durch das Psychotherapeutengesetz wurden ein neuer Berufsstand etabliert und Strukturen aus anderen akademischen Heilberufen auf ihn übertragen. Hierzu gehören die staatlich geregelte Ausbildung mit Staatsprüfung, Approbation, Integration in das System der Kassenärztlichen Vereinigung sowie die Selbstadministration und Qualitätssicherung innerhalb der Kammern. Durch die gesetzliche Fundierung und die Etablierung der unterschiedlichen Rahmenbedingungen hat der Beruf der Psychologischen Psychotherapeuten und der Kinder- und Jugendlichenpsychotherapeuten eine Identität bekommen, die deren Tätigkeit und deren Mitglieder deutlich aus dem Feld des «Psycho-Hokuspokus» heraushebt.

Besonders durch den Wissenschaftlichen Beirat Psychotherapie hat die Profession im Gesundheitssystem und in Ihrem Image nach Außen eine Möglichkeit erhalten, die Basisqualität ihrer therapeutischen Angebote zu sichern und zu dokumentieren. Durch die Betonung der (empirisch zu begründenden) Wissenschaftlichkeit ist den Psychologischen Instituten als universitären Einrichtungen für Forschung und Lehre die Aufgabe der Entwicklung bzw. Weiterentwicklung psychotherapeutischer Verfahren neu zugedacht worden. Der Gesetzgeber hat diese Aufgabe dadurch formuliert, dass zum Zweck der Forschung und Lehre Psychotherapie-Ambulanzen eingerichtet werden können (und sollen!).

Das Psychotherapeutengesetz hat jedoch auch eine Reihe un- günstiger, ungerechter oder schlecht gelöster Regelungen geschaffen. Im Unterschied zur Finanzierung der Ausbildung im medizinischen Heilberuf müssen angehende Psychologische Psychotherapeuten und Kinder- und Jugendlichenpsychotherapeuten trotz akademischem Abschluss ihre weit reichenden Kompetenzen im Rahmen des während ihrer Psychotherapieausbildung geforderten Praktikums in Kliniken meist unentgeltlich zur Verfügung stellen. Zusätzlich muss die Ausbildung von den Kandidaten finanziert werden. Auch werden die im Studium erworbenen Kenntnisse und Fertigkeiten im Rahmen der Ausbildung nicht anerkannt.

Eine wichtige, vor uns liegende Aufgabe ist die Sicherung des Nachwuchses und der psychotherapeutischen Versorgung der Bevölkerung, die sich an empirisch und epidemiologisch fundierten Zahlen zur Prävalenz psychischer Störungen orientiert und nicht rein administrativ motiviert ist. Notwendig ist auch die Anpassung der sozialrechtlichen Regelungen im Rahmen der Psychotherapierichtlinien und -vereinbarungen jenseits historisch gewachsener Regelungen und Interessen; basierend auf versorgungsrelevanten Effektivitäts- und Effizienzstudien. Weiterhin ist eine bessere Verzahnung der akademischen Basisausbildung und der Psychotherapieausbildung wichtig sowie eine empirisch fundierte Sicherung der Qualität der Versorgung. Damit in Zusammenhang steht eine klare Verantwortung des Gesundheitssystems und der Profession, versorgungsrelevante Forschung im Bereich der Psychotherapie $\mathrm{zu}$ finanzieren und $\mathrm{zu}$ betreiben.

Wir können gespannt sein, wie die Entwicklung in den kommenden Jahren weitergeht. Ich bin sicher, wir sind auf einem guten Weg.

Thomas Fydrich, Heidelberg 